Amir Yarahmadi 1, 2, 3, Zohreh Mostafavi-Pour4, Mohammad-Hadi Saeed Modaghegh², Negar Azarpira ${ }^{3}$, Alireza Mousavian², Shokoufeh Bonakdaran ${ }^{5}$, Fatemeh Sadeghipour Kermani ${ }^{2}$, Daryoush Hamidi Alamdari ${ }^{1,2}$

${ }^{1}$ Department of Clinical Biochemistry, Faculty of Medicine, Mashhad University of Medical Sciences, Mashhad, Iran ${ }^{2}$ Vascular and Endovascular Surgery Research Center, Mashhad University of Medical Sciences, Mashhad, Iran

${ }^{3}$ Transplant Research Center, Shiraz University of Medical Sciences, Shiraz, Iran

${ }^{4}$ Department of Biochemistry, School of Medicine, Shiraz University of Medical Sciences, Shiraz, Iran

${ }^{5}$ Metabolic Syndrome Research Center, Mashhad University of Medical Sciences, Mashhad, Iran

\title{
Association between serum vitamin $D$, hs-CRP, and prooxidant-antioxidant balance with anthropometric and biochemical parameters in patients with diabetic foot ulcers
}

\section{ABSTRACT}

Background. Oxidative balance and inflammatory processes affect wound healing phases, and their disruption is connected with delayed wound healing. The present study aimed to assess the association between serum hs-CRP, prooxidant-antioxidant Balance (PAB), and vitamin $D$ with anthropometric and biochemical parameters in patients with diabetic foot ulcers (DFU). Methods. Thirty-two patients with DFU were included in this study. The Spearman correlation coefficient was used to evaluate the bivariate relationship between serum hs-CRP, PAB, and vitamin D with anthropometric characteristics, glycemic status, lipid profiles, homocysteine level, liver, and kidney function tests.

Results. Our data showed a significant positive association between serum hs-CRP and insulin ( $r=0.417$, $P=0.027)$, uric acid $(r=0.629, P=0.001)$, creatinine

\section{Address for correspondence:}

Amir Yarahmadi and Zohreh Mostafavi-Pour are equally co-first authors. Dr Daryoush Hamidi Alamdari

Vascular and Endovascular

Surgery Research Center

Department of Clinical Biochemistry

Faculty of Medicine

Mashhad University of Medical Sciences, Iran

Phone/Fax: +987132343848

e-mail: hamidiad@mums.ac.ir

Clinical Diabetology 2020, 10, 1: 138-143

DOI: $10.5603 /$ DK.2020.0064

Received: 17.08 .2020

Accepted: 03.11.2020 $(r=0.431, P=0.022)$, erythema $(r=0.36, P=0.049)$, and ESR ( $r=0.560, P=0.002)$. Moreover, hs-CRP negatively correlated with FBS $(r=-0.427, P=0.023)$, total bilirubin $(r=-0.639, P=0.001)$, direct bilirubin $(r=-0.445, P=0.033)$, LDL-cholesterol $(r=-0.405$, $P=-0.032), B M I(r=-0.398, P=0.033)$ and HTN $(r=-0.450, P=0.014)$. Serum $P A B$ value negatively correlated with patients age $(r=-0.460, P=0.027)$, and BMI ( $r=-0.442, P=0.035)$, and positively associated with insulin level $(r=0.431, P=0.040)$. A significant positive association between serum vitamin $D$ with patient sex $(r=0.379, P=0.047)$, and QUICKI $(r=0.456, P=0.029)$, and negative correlation with $\mathrm{HbA}_{1 \mathrm{c}}(r=-0.381, \mathrm{P}=0.045)$ were also determined.

Conclusions. This study demonstrated that serum hs$C R P, P A B$, and vitamin D are significantly associated with some anthropometric and biochemical parameters with important clinical value in patients with DFU. Low levels of vitamin $D$ and high levels of hs-CRP and PAB may have an important role in the pathogenesis of DFU. (Clin Diabetol 2020; 10, 1: 138-143)

Key words: association, vitamin D, hs-CRP, oxidant-antioxidant balance, diabetic foot ulcers

\section{Introduction}

Diabetes is a multifactorial disease with a global epidemic underway [1]. It has been estimated that the 
number of patients with diabetes will increase to more than 380 million by the year 2025 [2]. The development of diabetes is associated with a wide range of complications, such as micro and macrovascular damages, which lead to diabetic foot ulcers (DFU) [3]. DFU is one of the most catastrophic diabetes complications associated with a high rate of lower extremity amputation among diabetic patients [4]. The exact mechanism by which diabetes causes these complications is very complex and has not been fully understood until date. This complication is connected to the direct effect of high blood glucose in diabetes. However, the indirect effect of oxidative stress, abnormal lipid profiles, chronic inflammatory condition, and low level of vitamin D may be involved in the progression of DFU [5-7].

Oxidative stress results from an imbalance between oxidant and antioxidant mechanisms within the body. Oxidative stress, through the generation of reactive oxygen/nitrogen radicals, attacks different components of cells such as proteins, carbohydrates, nucleic acids, and lipid membranes $[8,9]$. Assessment of prooxidant-antioxidant balance could be a helpful marker of oxidative status to make the right decision for proper medical treatment of diabetes.

Furthermore, it has been proved that high glucose in diabetes through the formation of advanced glycation end-products (AGEs) increases inflammation, resulting in worsening disease condition and its complications [10]. Among different inflammation markers, C-reactive protein (CRP), an acute-phase reactant protein, has been regarded as a sensitive marker of systemic inflammation [11]. Interleukin-1 (IL-1), IL-6, and tumor necrosis factor-alpha (TNF- $\alpha$ ) regulate CRP production within the liver [12]. Lately, more sensitive immunoassays for CRP measurement (high sensitivity CRP, hs-CRP) have become available and made this possible to measure the low quantity of CRP and compare it with other inflammatory parameters in the blood [13]. Previous studies revealed a relationship between hs-CRP levels and the development and progression of coronary heart disease (CHD) and osteoarthritis $[14,15]$. PAB is a simple assay, which measures total prooxidants and antioxidants within one assay. It makes it possible to understand the role of oxidative stress in the pathophysiology of many diseases such as diabetes and its complications such as DFU [16].

Moreover, previous studies found that a low level of vitamin $D$ is a possible risk factor for type 2 diabetes. Interestingly, they have shown an inverse relationship between serum vitamin $D$ and the incidence of DFU [17]. Therefore, in the present study, we aimed to evaluate the association of serum vitamin D, hs-CRP, and oxidative balance with anthropometric characteristics, glycemic status, lipid profiles, homocysteine level, liver, and kidney function tests in patients with DFU.

\section{Methods}

Patients

This study was performed among thirty-two patients with DFU referred to the Alavi Vascular Surgery Hospital in Mashhad, Iran. The ethics committee of the Mashhad University of Medical Sciences approved the study protocol (registration number: IR.MUMS. REC.1398.251). Written informed consent was obtained from each participant.

\section{Clinical measurements}

After an initial assessment of disease conditions, anthropometric measurements were obtained. Following overnight fasting, patients' weight was determined by a standard scale (EmsiG, Hamburg, Germany). The participants' height was measured using a nonstretched tape measure (EmsiG, Hamburg, Germany) to the nearest $0.1 \mathrm{~cm}$. Then, BMI was calculated as body weight in kilograms divided by height in meters squared. All patients answered the required clinical and epidemiological assessment evaluating the treatment with insulin, metformin, and other oral hypoglycemic drugs, hypertension, time of diabetes diagnosis, and the development of any chronic complications related to diseases. All data were confirmed by a review of the patient's medical records.

\section{Laboratory analysis}

Venous blood samples $(10 \mathrm{~mL})$ were taken from all patients following overnight fasting. Then, blood was centrifuged at $2500 \times \mathrm{g}$ for $15 \mathrm{~min}$ at $4{ }^{\circ} \mathrm{C}$ to separate serum. Serum vitamin $D$ was determined using a commercial ELISA kit (ZellBio, Veltlinerweg, Germany). Serum hs-CRP was measured using a commercial ELISA kit (ZellBio, Veltlinerweg, Germany) with a microplate reader (Rosys Anthos 2010, Wals, Austria). Serum prooxidant-antioxidant balance (PAB) was measured based on a previous method developed by Hamidi Alamdari [18]. Serum ESR was obtained by the Westergren methods [19]. Homocysteine was measured by a commercial ELISA kit (Aviva, California, USA). $\mathrm{HbA}_{1 \mathrm{c}}$ levels were evaluated by the Glycomat kit (BiocodeHycel, Massy, France). Serum insulin concentrations were obtained by a commercial ELISA kit (Mercodia, Uppsala, Sweden). All the inter- and intra-assay CVs for serum vitamin $\mathrm{D}$, hs-CRP, homocysteine, $\mathrm{HbA}_{1 c^{\prime}}$ and insulin concentrations were less than $5 \%$. The homeostasis model of assessment-insulin resistance (HOMA-IR) and the quantitative insulin sensitivity check index (QUICKI) were calculated based on the suggested formulas [20]. 
Table 1. Anthropometric and clinical characteristics of the study participants

\begin{tabular}{lc}
\hline Trait value & \\
\hline Age (year) & $58.5 \pm 9.4$ \\
Sex (male/female) & $20 / 12$ \\
Diabetes duration (year) & $12.4 \pm 7.1$ \\
Type 2 diabetes (\%) & $32(100)$ \\
Insulin injection (\%) & $21(65.6)$ \\
Metformin therapy (\%) & $18(56.2)$ \\
Glibenclamide therapy (\%) & $6(18.7)$ \\
HTN (\%) & $17(53.1)$ \\
Retinopathy (\%) & $19(59.3)$ \\
Previous amputation (\%) & $12(37.5)$ \\
Ulcer symptoms & \\
Erythema (\%) & $22(68.7)$ \\
Discharge (\%) & $14(43.8)$ \\
Necrosis (\%) & $11(34.3)$ \\
Height & $175.6 \pm 5.5$ \\
Weight & $77.1 \pm 11.6$ \\
BMI & $25.1 \pm 3.8$ \\
\hline
\end{tabular}

All values are presented as means $\pm \mathrm{SDs} ; \mathrm{BMI}$ - body mass index; HTN - hypertension

We have used Enzymatic kits to evaluate serum fasting blood sugar (FBS), triglycerides (TG), urea, creatinine, uric acid, cholesterol (Chol), VLDL-Chol, LDL-Chol, HDL-Chol, total bilirubin, alkaline phosphatase (ALP), alanine aminotransferase (ALT), and aspartate aminotransferase (AST) according to the manufacturer protocols (Pars Azmun, Tehran, Iran). All the inter- and intra-assay CVs for serum FBS, urea, creatinine, uric acid, bilirubin, ALP, ALT, AST, and lipid profiles were less than $5 \%$. Albumin concentrations were obtained by a commercial ELISA kit (Pars Azmun, Tehran, Iran).

\section{Statistical analysis}

Data are presented as the mean \pm standard deviation (SD). The Spearman correlation coefficient was used to evaluate the bivariate relationship between serum levels of vitamin D, hs-CRP, and PAB with anthropometric characteristics, glycemic status, lipid profiles, homocysteine level, liver, and kidney function tests. $\mathrm{P}<0.05$ was considered as the level of significance. All statistical analyses were performed using the SPSS 22 statistical package (SPSS22, Chicago, IL, USA).

\section{Results}

The baseline anthropometric and clinical characteristics of study subjects are presented in Table 1. This study was performed among thirty-two patients with DFU, in which 21 patients $(65.6 \%)$ had at least one
Table 2. Baseline biochemical parameters of the study participants

\begin{tabular}{|c|c|}
\hline Parameter value & \\
\hline FBS [mg/dL] & $141.2 \pm 39.7$ \\
\hline Insulin $[\mu \mathrm{IU} / \mathrm{mL}]$ & $14.9 \pm 12.5$ \\
\hline HOMA-IR & $5.0 \pm 3.9$ \\
\hline QUICKI & $0.31 \pm 0.03$ \\
\hline $\mathrm{HbA}_{1 \mathrm{c}}(\%)$ & $9.8 \pm 1.7$ \\
\hline Uric acid [mg/dL] & $5.3 \pm 1.5$ \\
\hline Urea [mg/dL] & $40.7 \pm 22.1$ \\
\hline Creatinine [mg/dL] & $1.0 \pm 0.37$ \\
\hline Triglycerides [mg/dL] & $120.6 \pm 59.0$ \\
\hline Total cholesterol [mg/dL] & $147.6 \pm 35.2$ \\
\hline VLDL cholesterol [mg/dL] & $24.0 \pm 11.7$ \\
\hline LDL cholesterol [mg/dL] & $74.4 \pm 27.9$ \\
\hline HDL cholesterol [mg/dL] & $2.1 \pm 16.7$ \\
\hline Total-/HDL-cholesterol ratio & $3.8 \pm 1.1$ \\
\hline Bilirubin total [mg/dL] & $0.45 \pm 0.37$ \\
\hline Bilirubin direct $[\mathrm{mg} / \mathrm{dL}]$ & $0.20 \pm 0.09$ \\
\hline ALP [IU/L] & $189.3 \pm 69.4$ \\
\hline AST [IU/L] & $19.6 \pm 4.7$ \\
\hline ALT [IU/L] & $18.0 \pm 7.9$ \\
\hline Albumin [g/dL] & $4.3 \pm 0.41$ \\
\hline Homocysteine $[\mu \mathrm{mol} / \mathrm{L}]$ & $9.9 \pm 3.4$ \\
\hline Vitamin D3 [ng/mL] & $25.3 \pm 20.0$ \\
\hline $\operatorname{ESR}[\mathrm{mm} / \mathrm{h}]$ & $41.4 \pm 38.5$ \\
\hline hs-CRP [mg/L] & $24.7 \pm 29.4$ \\
\hline PAB (HK unit) & $169.5 \pm 47.4$ \\
\hline
\end{tabular}

All values are presented as means \pm SDs; BMI — body mass index; HTN hypertension; FBS - fasting blood sugar; HOMA-IR - homeostasis model of assessment-insulin resistance; QUICKI - quantitative insulin sensitivity check index; $\mathrm{HbA}_{1 \mathrm{c}}$ - hemoglobin $\mathrm{A}_{1 c^{\prime}}$ hs-CRP — high-sensitivity C-reactive protein; ESR - erythrocyte sedimentation rate; ALP — alkaline phosphatase; AST — aspartate aminotransferase; ALT — alanine aminotransferase; $\mathrm{PAB}$ - prooxidant-antioxidant balance

insulin daily injection, and 18 patients (56.2\%) used oral metformin. 17 patients (53.1\%) had a history of hypertension (HTN) and consumed oral antihypertensive drugs. The mean BMI of the study participants was $25.1 \pm 3.8$.

Biochemical parameters of study subjects are listed in Table 2. As shown in Table 3, the correlation coefficient revealed that the serum levels of hs-CRP was positively associated with insulin $(r=0.417$, $P=0.027)$, uric acid $(r=0.629, P=0.001)$, creatinine $(r=0.431, P=0.022)$, erythema $(r=0.36, P=0.049)$, and $\operatorname{ESR}(r=0.560, P=0.002)$. Also, our results showed that hs-CRP levels negatively correlated with BMI $(r=-0.398, P=0.033)$, HTN $(r=-0.450, P=0.014)$, FBS $(r=-0.427, P=0.023)$, total bilirubin $(r=-0.639$, $P=0.001)$, direct bilirubin $(r=-0.445, P=0.033)$ and LDL-cholesterol $(r=-0.405, P=-0.032)$. There was no 
Table 3. Correlation between serum hs-CRP, PAB, and vitamin D with anthropometric and biochemical parameters in patients with DFU

\begin{tabular}{|c|c|c|c|c|c|c|}
\hline & \multicolumn{2}{|c|}{ Serum hs-CRP values } & \multicolumn{2}{|c|}{ Serum PAB values } & \multicolumn{2}{|c|}{ Serum Vitamin D } \\
\hline & $r$ values & $P$ values & $r$ values & $P$ values & r values & $P$ values \\
\hline Age & -0.286 & 0.131 & -0.460 & 0.027 & 0.284 & 0.143 \\
\hline Sex & -0.121 & 0.530 & 0.299 & 0.166 & 0.379 & 0.047 \\
\hline Diabetes duration (year) & -0.073 & 0.708 & -0.056 & 0.799 & 0.112 & 0.571 \\
\hline Height & 0.045 & 0.816 & 0.355 & 0.096 & 0.281 & 0.147 \\
\hline Weight & -0.344 & 0.068 & -0.293 & 0.175 & 0.037 & 0.851 \\
\hline BMI & -0.398 & 0.033 & -0.442 & 0.035 & -0.036 & 0.856 \\
\hline HTN & -0.450 & 0.014 & -0.013 & 0.953 & -0.027 & 0.893 \\
\hline FBS $[\mathrm{mg} / \mathrm{dL}]$ & -0.427 & 0.023 & -0.230 & 0.290 & -0.230 & 0.239 \\
\hline Insulin $[\mu \mathrm{IU} / \mathrm{mL}]$ & 0.417 & 0.027 & 0.431 & 0.040 & -0.081 & 0.682 \\
\hline HOMA-IR & 0.196 & 0.317 & 0.247 & 0.257 & -0.314 & 0.104 \\
\hline QUICKI & -0.205 & 0.296 & -0.245 & 0.260 & 0.456 & 0.029 \\
\hline $\mathrm{HbA}_{1 \mathrm{c}}(\%)$ & 0.001 & 0.998 & 0.410 & 0.052 & -0.381 & 0.045 \\
\hline Uric Acid [mg/dL] & 0.629 & 0.001 & 0.224 & 0.304 & -0.018 & 0.929 \\
\hline Urea [mg/dL] & 0.147 & 0.456 & -0.171 & 0.435 & -0.221 & 0.259 \\
\hline Creatinine $[\mathrm{mg} / \mathrm{dL}]$ & 0.431 & 0.022 & -0.070 & 0.750 & -0.029 & 0.882 \\
\hline Triglycerides [mg/dL] & 0.001 & 0.999 & -0.055 & 0.802 & -0.149 & 0.449 \\
\hline Total cholesterol [mg/dL] & 0.344 & 0.073 & 0.113 & 0.607 & -0.044 & 0.825 \\
\hline VLDL cholesterol [mg/dL] & -0.001 & 0.997 & -0.051 & 0.817 & -0.166 & 0.399 \\
\hline LDL cholesterol [mg/dL] & -0.405 & 0.032 & 0.037 & 0.867 & -0.014 & 0.945 \\
\hline HDL cholesterol [mg/dL] & 0.265 & 0.173 & -0.039 & 0.861 & -0.038 & 0.848 \\
\hline Total-/HDL-cholesterol ratio & 0.060 & 0.786 & 0.015 & 0.946 & -0.089 & 0.687 \\
\hline Bilirubin total [mg/dL] & -0.639 & 0.001 & 0.081 & 0.713 & 0.149 & 0.448 \\
\hline Bilirubin direct $[\mathrm{mg} / \mathrm{dL}]$ & -0.445 & 0.033 & -0.354 & 0.14 & 0.140 & 0.523 \\
\hline ALP [IU/L] & 0.362 & 0.058 & 0.191 & 0.383 & 0.001 & 0.998 \\
\hline AST [IU/L] & -0.095 & 0.629 & 0.017 & 0.937 & 0.037 & 0.853 \\
\hline ALT [IU/L] & -0.259 & 0.184 & 0.104 & 0.636 & 0.040 & 0.839 \\
\hline Albumin [g/dL] & -0.265 & 0.174 & 0.232 & 0.286 & 0.143 & 0.467 \\
\hline Homocysteine $[\mu \mathrm{mol} / \mathrm{L}]$ & 0.202 & 0.303 & 0.267 & 0.218 & 0.004 & 0.983 \\
\hline Vitamin D3 [ng/mL] & -0.010 & 0.958 & 0.263 & 0.226 & - & - \\
\hline $\mathrm{ESR}[\mathrm{mm} / \mathrm{h}]$ & 0.560 & 0.002 & 0.061 & 0.781 & -0.032 & 0.871 \\
\hline hs-CRP [mg/L] & - & - & 0.281 & 0.194 & -0.010 & 0.958 \\
\hline PAB (HK unit) & 0.281 & 0.194 & - & - & 0.263 & 0.226 \\
\hline
\end{tabular}

$r$ value represent Spearman correlation coefficient; $\mathrm{P}$ values represent Spearman correlation test

All values are presented as means \pm SDs; BMI — body mass index; HTN - hypertension; FBS - fasting blood sugar; HOMA-IR - homeostasis model of assessment-insulin resistance; QUICKI - quantitative insulin sensitivity check index; $\mathrm{HbA}_{1 \mathrm{c}}$ - hemoglobin $\mathrm{A}_{1 \mathrm{c}}$; hs-CRP — high-sensitivity C-reactive protein; ESR - erythrocyte sedimentation rate; ALP — alkaline phosphatase; AST — aspartate aminotransferase; ALT — alanine aminotransferase; PAB — prooxidant-antioxidant balance

significant correlation between hs-CRP level and age, sex, height, weight, other biochemical parameters including TG, cholesterol, albumin, vitamin D3, homocysteine, PAB and liver function tests.

A significant and negative associations were found between $P A B$ and patients age $(r=-0.460, P=0.027)$, $\operatorname{BMI}(r=-0.442, P=0.035)$, and a positive correlation of PAB with insulin $(r=0.431, P=0.040)$ was also observed. Moreover, a positive and marginal association between $\mathrm{PAB}$ value and $\mathrm{HbA}_{1 \mathrm{c}}$ were seen $(r=0.410$,
$P=0.052)$ in patients with DFU. Our data showed that there was not any significant correlation between PAB value and other anthropometric and biochemical parameters, as shown in Table 3.

Vitamin $D$ positively associated with sex $(r=0.379$, $P=0.047)$ and QUICKI $(r=0.456, P=0.029)$. Furthermore, a negative correlation between serum vitamin $\mathrm{D}$ and $\mathrm{HbA}_{1 \mathrm{c}}(\mathrm{r}=-0.381, \mathrm{P}=0.045)$ were also determined (Table 3 ). There was no significant association between serum vitamin $\mathrm{D}$, anthropometric 

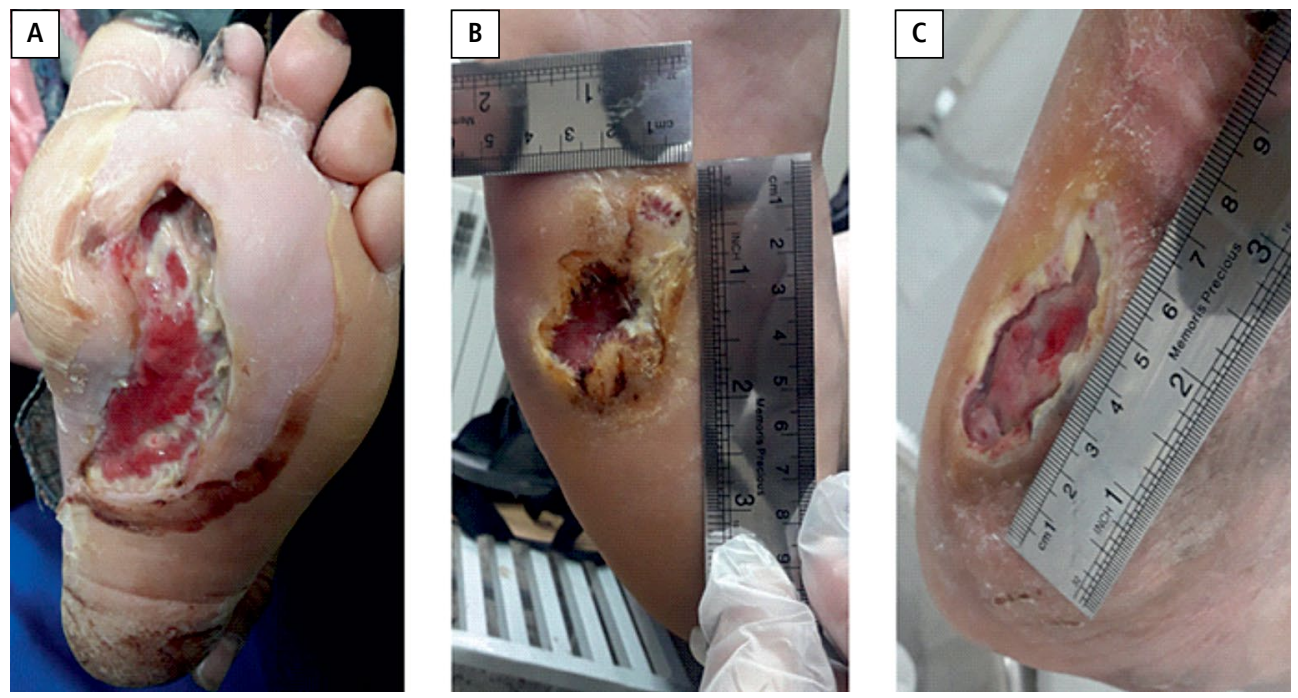

Figure 1. Three patients with DFU; A - a 54 years old woman with DFU for 3 months; $\mathbf{B}$ - a 49 years old woman with DFU for 18 months; C - a 51 years old man with DFU for 12 months

characteristics and other biochemical parameters as documented in Table 3.

\section{Discussion}

The present study results showed that the hs-CRP level is associated with glycemic (FBS and insulin), oxidative, and inflammatory markers in patients with DFU. Similar reports were published by some investigators, which showed that hyperglycemia promotes oxidative and inflammatory markers in patients with diabetes [21]. CRP, a member of acute-phase reactant proteins, has been used widely as a marker of cardiovascular complications [22]. It has been suggested that CRP has direct pro-inflammatory effects, and assessment of CRP (as hs-CRP) could be a valid marker for evaluating inflammatory problems during diseases [[23. Similar to our study, Zubair et al. [24] showed a correlation between hs-CRP and BMI, LDL-cholesterol, and nephropathy in patients with DFU. In our study, we saw a significant correlation between hs-CRP and creatinine level, which is the best marker of diabetic nephropathy. Numerous data demonstrated that inflammation plays an important role in the pathophysiology of DFU [25]. Also, it has been proven that insulin sensitivity or insulin resistance is an inflammatory process [26], as shown in our study that hs-CRP had a positive correlation with insulin and ESR level. Moreover, we found a significant and negative correlation between $\mathrm{PAB}$ and age $(r=$ $=-0.460, P=0.027)$, and $\mathrm{BMI}(r=-0.442, \mathrm{P}=0.035)$; however, there was no significant association between PAB and other inflammatory and biochemical markers in serum of patients with DFU. Besides, we did not find any significant correlation between hs-CRP and PAB with homocysteine and vitamin D level in DFU patients. Consistent with our results, Jung et al. [27] showed that serum vitamin $D$ level positively correlated with a patient's sex. Also, our data revealed a significant negative correlation between serum vitamin $\mathrm{D}$ and $\mathrm{HbA}_{1 \mathrm{c}}$. Similar to our study Buhary et al. [28] also found that $\mathrm{HbA}_{1 \mathrm{c}}$ was inversely related to serum vitamin $\mathrm{D}$ levels $(r=-0.14, P<0.001)$ [28]. A meta-analysis by Lee et al. [29] showed that vitamin D supplementation resulted in a modest reduction of $\mathrm{HbA}_{1 \mathrm{c}}$ in type2 diabetic patients. In summary, we found a correlation between hs-CRP and glycemic, oxidative, and inflammatory markers in patients with DFU. Furthermore, there was a significant correlation between vitamin $\mathrm{D}$ and $\mathrm{HBA}_{1 \mathrm{c}}$ level, and there was no correlation between vitamin $D$ and other biochemical parameters. A high level of hsCRP may predict the progression of diabetes and may have an important role in the pathogenesis of diabetes complications. We had a few limitations in our study; it was a single-center study with relatively small sample size. A multicenter study involving a higher number of patients is recommended.

\section{Conclusions}

In conclusion, the results of this study demonstrate that serum hs-CRP, PAB, and vitamin D are significantly associated with some anthropometric and biochemical parameters with important clinical value in patients with DFU. Low levels of vitamin D and high levels of hs-CRP and PAB may have an important role in the pathogenesis of DFU. 


\section{Acknowledgments}

This study has been extracted from the PhD thesis of Amir Yarahmadi, conducted in Vascular and Endovascular Surgery Research Center of Mashhad University of Medical Sciences, Mashhad, Iran.

\section{Funding}

This work was supported by the Research Council at Mashhad University of Medical Sciences under Grant number 971636.

\section{Conflict of interest}

The authors declare that they have no conflict of interest related to this manuscript.

\section{REFERENCES}

1. Zimmet P, Alberti KG, Shaw J. Global and societal implications of the diabetes epidemic. Nature. 2001; 414(6865): 782-787, doi: 10.1038/414782a, indexed in Pubmed: 11742409.

2. Zimmet P, Alberti KG, Magliano DJ, et al. Diabetes mellitus statistics on prevalence and mortality: facts and fallacies. Nat Rev Endocrinol. 2016; 12(10): 616-622, doi: 10.1038/nrendo.2016.105, indexed in Pubmed: 27388988.

3. Dee T, Sukartini T, Probowati R. Factors associated with foot ulcer among diabetic patients. Int J Nurs Sci Health Serv. 2020; 3(3): 771-776.

4. Martins-Mendes D, Monteiro-Soares M, Boyko EJ, et al. The independent contribution of diabetic foot ulcer on lower extremity amputation and mortality risk. J Diabetes Complications. 2014; 28(5): 632-638, doi: 10.1016/j.jdiacomp.2014.04.011, indexed in Pubmed: 24877985.

5. Singh SK, Sahay RK, Krishna A. Oxidative stress in diabetic foot ulcer. Diabetes \& Metabolic Syndrome: Clinical Research \& Reviews. 2008; 2(2): 109-113, doi: 10.1016/j.dsx.2008.02.003.

6. Zubair M, Malik A, Meerza D, et al. 25-Hydroxyvitamin D [25(OH) D] levels and diabetic foot ulcer: is there any relationship? Diabetes Metab Syndr. 2013; 7(3): 148-153, doi: 10.1016/j. dsx.2013.06.008, indexed in Pubmed: 23953180.

7. Yarahmadi A, Zal F, Bolouki A. Protective effects of quercetin on nicotine induced oxidative stress in 'HepG2 cells'. Toxicol Mech Methods. 2017; 27(8): 609-614, doi: 10.1080/15376516.2017.1344338, indexed in Pubmed: 28627253.

8. Sies H, Berndt C, Jones DP. Oxidative stress. Annu Rev Biochem. 2017; 86: 715-748, doi: 10.1146/annurev-biochem-061516-045037, indexed in Pubmed: 28441057

9. Yarahmadi A, Khademi F, Mostafavi-Pour Z, et al. In-vitro analysis of glucose and quercetin effects on $\mathrm{m}$-TOR and Nrf-2 expression in HepG2 cell line (diabetes and cancer connection). Nutr Cancer. 2018; 70(5): 770-775, doi: 10.1080/01635581.2018.1470654, indexed in Pubmed: 29781726.

10. Khan R, Ooi XY, Parvus M, et al. Advanced glycation end products: formation, role in diabetic complications, and potential in clinical applications. The Eye and Foot in Diabetes. 2020, doi: 10.5772/ intechopen.89408.

11. Sproston NR, Ashworth JJ. Role of C-reactive protein at sites of inflammation and infection. Front Immunol. 2018; 9: 754, doi: 10.3389/fimmu.2018.00754, indexed in Pubmed: 29706967

12. Ahmad J, Zubair M, Malik A, et al. Cathepsin-D, adiponectin, TNF- $\alpha$, IL- 6 and hsCRP plasma levels in subjects with diabetic foot and possible correlation with clinical variables: a multicentric study. Foot (Edinb). 2012; 22(3): 194-199, doi: 10.1016/j. foot.2012.03.015, indexed in Pubmed: 22560191.
13. Windgassen EB, Funtowicz L, Lunsford TN, et al. C-reactive protein and high-sensitivity C-reactive protein: an update for clinicians. Postgrad Med. 2011; 123(1): 114-119, doi: 10.3810/ pgm.2011.01.2252, indexed in Pubmed: 21293091.

14. Rifai N, Ridker P. High-sensitivity C-reactive protein: a novel and promising marker of coronary heart disease. Clinical Chemistry. 2001; 47(3): 403-411, doi: 10.1093/clinchem/47.3.403.

15. Pearle AD, Scanzello CR, George $S$, et al. Elevated high-sensitivity $C$-reactive protein levels are associated with local inflammatory findings in patients with osteoarthritis. Osteoarthritis Cartilage. 2007; 15(5): 516-523, doi: 10.1016/j.joca.2006.10.010, indexed in Pubmed: 17157039.

16. Hamidi Alamdari D, Bagheri Moghaddam A, Amini S, et al. The application of a reduced dye used in orthopedics as a novel treatment against coronavirus (COVID-19): a suggested therapeutic protocol. Arch Bone Jt Surg. 2020; 8(Suppl1): 291-294, doi: 10.22038/abjs.2020.47745.2349, indexed in Pubmed: 32607398.

17. Dai J, Jiang $C$, Chen $H$, et al. Vitamin $D$ and diabetic foot ulcer: a systematic review and meta-analysis. Nutr Diabetes. 2019; 9(1): 8, doi: 10.1038/s41387-019-0078-9, indexed in Pubmed: 30858355.

18. Alamdari DH, Paletas $K$, Pegiou $T$, et al. A novel assay for the evaluation of the prooxidant-antioxidant balance, before and after antioxidant vitamin administration in type II diabetes patients. Clin Biochem. 2007; 40(3-4): 248-254, doi: 10.1016/j. clinbiochem.2006.10.017, indexed in Pubmed: 17196578.

19. Sikka M, Tandon R, Rusia U, et al. Validation of ESR analyzer using Westergren ESR method. Indian J Pathol Microbiol. 2007; 50: 634.

20. Borai A, Livingstone C, Kaddam I, et al. Selection of the appropriate method for the assessment of insulin resistance. BMC Med Res Methodol. 2011; 11: 158, doi: 10.1186/1471-2288-11-158, indexed in Pubmed: 22112229.

21. Aronson D. Hyperglycemia and the pathobiology of diabetic complications. Adv Cardiol. 2008; 45: 1-16, doi: 10.1159/000115118, indexed in Pubmed: 18230953.

22. Yeh ET, Anderson HV, Pasceri V, et al. C-reactive protein: linking inflammation to cardiovascular complications. Circulation. 2001; 104(9): 974-975, doi: 10.1161/01.cir.104.9.974, indexed in Pubmed: 11524386.

23. Liang YJ, Liu YC, Chen CY, et al. Comparison of PPAR $\delta$ and PPAR $\gamma$ in inhibiting the pro-inflammatory effects of C-reactive protein in endothelial cells. Int J Cardiol. 2010; 143(3): 361-367, doi: 10.1016/j.ijcard.2009.03.100, indexed in Pubmed: 19395102.

24. Zubair M, Malik A, Ahmad J. Plasma adiponectin, IL-6, hsCRP, and TNF- levels in subject with diabetic foot and their correlation with clinical variables in a North Indian tertiary care hospital. Indian J Endocrinol Metab. 2012; 16(5): 769-776, doi: 10.4103/22308210.100672, indexed in Pubmed: 23087862.

25. Mi Qi, Rivière $B$, Clermont $G$, et al. Agent-based model of inflammation and wound healing: insights into diabetic foot ulcer pathology and the role of transforming growth factor-beta 1. Wound Repair Regen. 2007; 15(5): 671-682, doi: 10.1111/j.1524475X.2007.00271.x, indexed in Pubmed: 17971013

26. Hotamisligil GS. Inflammatory pathways and insulin action. Int J Obes Relat Metab Disord. 2003; 27 Suppl 3: S53-S55, doi: 10.1038/sj.ijo.0802502, indexed in Pubmed: 14704746.

27. Jung $\mathrm{CH}$, Kim KJ, Kim BY, et al. Relationship between vitamin D status and vascular complications in patients with type 2 diabetes mellitus. Nutr Res. 2016; 36(2): 117-124, doi: 10.1016/j. nutres.2015.11.008, indexed in Pubmed: 26826427.

28. Buhary BM, Almohareb O, Aljohani N, et al. Association of glycosylated hemoglobin levels with vitamin D status. J Clin Med Res. 2017; 9(12): 1013-1018, doi: 10.14740/jocmr3227w, indexed in Pubmed: 29163736.

29. Lee CJ, lyer G, Liu Y, et al. The effect of vitamin D supplementation on glucose metabolism in type 2 diabetes mellitus: A systematic review and meta-analysis of intervention studies. J Diabetes Complications. 2017; 31(7): 1115-1126, doi: 10.1016/j. jdiacomp.2017.04.019, indexed in Pubmed: 28483335. 